PHYSICAL REVIEW B 72, 099902(E) (2005)

\title{
Erratum: Classical theory of the ground spin-state in cubic spinels [Phys. Rev. 126, 540 (1962)]
}

D. H. Lyons, T. A. Kaplan, K. Dwight, and N. Menyuk

(Received 4 August 2005; published 28 September 2005)

DOI: 10.1103/PhysRevB.72.099902

PACS number(s): 75.10.-b, 75.25.+z, 99.10.Cd

In Eq. (B2), there are misprints in the third terms of $\eta_{1}$ and $\eta_{2}$ and the first term of $\eta_{4}$. The correct expressions for the $\eta^{\prime}$ s are

$$
\begin{aligned}
& \eta_{1}=\frac{1}{3}\{\exp [i \mathbf{k} \cdot(\overline{1} 13)]+\exp [i \mathbf{k} \cdot(31 \overline{1})]+\exp [i \mathbf{k} \cdot(\overline{1} \overline{3} \overline{1})]\} \\
& \eta_{2}=\frac{1}{3}\{\exp [i \mathbf{k} \cdot(1 \overline{1} 3)]+\exp [i \mathbf{k} \cdot(13 \overline{1})]+\exp [i \mathbf{k} \cdot(\overline{3} \overline{1} \overline{1})]\}, \\
& \eta_{3}=\frac{1}{3}\{\exp [i \mathbf{k} \cdot(1 \overline{1} \overline{3})]+\exp [i \mathbf{k} \cdot(\overline{3} 11)]+\exp [i \mathbf{k} \cdot(1 \overline{3} 1)]\}, \\
& \eta_{4}=\frac{1}{3}\{\exp [i \mathbf{k} \cdot(\overline{1} \overline{1} \overline{3})]+\exp [i \mathbf{k} \cdot(\overline{1} 31)]+\exp [i \mathbf{k} \cdot(3 \overline{1} 1)]\} .
\end{aligned}
$$

The remainder of the paper is unaffected by this correction. 